Open Access

\title{
Evaluation of multiplex ligation dependent probe amplification (MLPA) for identification of acute lymphoblastic leukemia with an intrachromosomal amplification of chromosome 21 (iAMP21) in a Brazilian population
}

Gerhard Fuka, Tállita M. Farias-Vieira, Leticia Hummel, Caroline B. Blunck, Júlio C. Santoro, Eugênia Terra-Granado, Thayana Conceição Barbosa, Mariana Emerenciano and Maria S. Pombo-de-Oliveira ${ }^{*}$

\begin{abstract}
Background: An intrachromosomal amplification of chromosome 21 (iAMP21) defines a unique subgroup of B-cell precursor acute lymphoblastic leukemia (BCP-ALL). The finding of three or more extra copies of the RUNX1 gene by fluorescence in situ hybridization (FISH) is internationally used to define an iAMP21. Genomic profiling of chromosome 21 has been suggested for assisting diagnostic case identification. Due to limitations of comparative genomic hybridization, in terms of a routine application as first line-screening tests we evaluated the multiplex ligation-dependent probe amplification (MLPA) SALSA P327_A1 and P327_B1 probe sets for detecting chromosome 21 copy number alterations in Brazilian childhood BCP-ALL.
\end{abstract}

Results: In 74 out of 368 patients gain of genetic material was detected. For data confirmation RUNX1 directed FISH was performed. Cells with $\geq 5$ RUNX1 signals $(n=9)$ were considered as "true iAMP21" while $<5$ RUNX1 signals $(n=41)$ were counted as evidence for additional copies of intact chromosomes 21. All patients with an iAMP21 had high MLPA peak ratios ( $\geq 1.8$ ), while the majority of patients with $<5 R U N X 1$ presented low MLPA peak ratios $(<1.8)$. Observed differences gained statistical strength by comparing probes located within the common region of amplification. Next, a principal component analysis was performed in order to illustrate distribution of cases according to their MLPA peak profile in two dimensions. Cases with an IAMP21 mostly clustered together, however additional cases with $<5$ RUNX1 signals or no available FISH data located in proximity.

Conclusions: MLPA qualified as a high throughput technique that could be employed in future studies for a critical comparison with data obtained by FISH, especially in cases where metaphase nuclei are not available. Taking submicroscopic aberrations into account examined by MLPA, cases exhibiting an "iAMP21 like" peak ratio profile but $<5$ RUNX1 signals should be considered as candidates for this chromosomal abnormality.

Keywords: B-cell precursor acute lymphoblastic leukemia (BCP-ALL), Intrachromosomal amplification of chromosome 21 (iAMP21), Multiplex ligation dependent probe amplification (MLPA), Fluorescence in situ hybridization (FISH)

\footnotetext{
* Correspondence: mpombo@inca.gov.br

Pediatric Hematology-Oncology Program, Research Center, Instituto Nacional de Câncer (INCA), Rua André Cavalcanti, 37, Rio de Janeiro, RJ 20231-050, Brazil
} 


\section{Background}

Cytogenetic and molecular studies have displayed among B-cell precursor-acute lymphoblastic leukemia (BCP-ALL) a distinct subtype, characterized by an intrachromosomal amplification of chromosome 21 (iAMP21) [1, 2].

Patients with an iAMP21 treated under standard risk regimens had an elevated relapse rate and a dismal prognosis compared to other BCP-ALL, hence risk directed treatment intensification has been recommended [3-7]. Amplification of 21q has also been found in other hematopoietic malignancies such as acute myeloid leukemia and disorders with complex karyotypes involving chromosome 21, supporting the hypothesis that gains within chromosome 21 interplay with the function of a specific set of genes [8]. Array based genomic analyses revealed that alterations on chromosome 21 are highly complex and suggested to arise from breakage-fusion bridge cycles followed by chromothripsis and other complex structural rearrangements on chromosome 21 [9-11]. Multiple regions of gains, amplifications, inversions and deletions with a high level of individuality between different patients have been described. Patients exhibited a common region of amplification (CRA, covering a region of $6 \mathrm{MB}$ ) as well as in most cases a common region of deletion (CRD, covering a region of $9 \mathrm{MB}$ ) [10]. The CRA, located between 32.8-37.9 MB on chromosome 21, consistently showed the most amplified as well as overexpressed regions, encoding for genes that play important roles in leukemia such as RUNX1, DYRK1A and ETS2 [11].

The method nowadays internationally recommended to identify an iAMP21 is fluorescence in situ hybridization (FISH) using probes directed to the Runt Related Transcription Factor 1 (RUNX1) gene, present by three or more extra copies on a single abnormal chromosome $21[10,12]$. However, case interpretation needs to be done cautiously, particularly whenever FISH was performed from interphase nuclei because additional copies of the RUNX1 genes are also present in high hyperdiploid karyotypes. In light of such considerations, genomic analyses of chromosome 21 might be taken into account for confirming the accuracy of iAMP21 diagnosis [13]. Multiplex ligation-dependent probe amplification (MLPA) assays represent a quick and cost effective alternative to array based techniques allowing screening of large cohorts for sub microscopic copy number changes. By quantification of multiple gene sequences on multiple sites on a previously defined chromosomal region such as an abnormal chromosome 21 it permits data comparison with other molecular techniques [14].

In this work, we evaluated MLPA for detecting an iAMP21 in a cohort of Brazilian childhood BCP-ALL and compared data with those obtained from FISH, conventionally detecting RUNX1 copy numbers. To our knowledge, this is the first published report where the SALSA MLPA
P327_A1 and P327_B1 probe sets were tested in order to identify copy number alterations (CNAs) in a subset of genes, demonstrating its value as a tool for screening large cohorts of patients.

\section{Results}

Demographic and clinical characteristics of 368 BCP-ALL alleged according to designated criteria with the MLPA results are shown in Table 1 . Most variables were equally distributed in negative and positive cases, but age between 2-10 years, common-ALL and standard prognostic risk was more prone in MLPA positive patients $(\mathrm{p}<0.05)$. Blast cell counts were similar in both groups with an average of $82 \%$ (range $67 \%$ to $90 \%$ ) in the whole cohort.

Table 1 Baseline characteristics of BCP-ALL with and without chromosome 21 CNAs

\begin{tabular}{|c|c|c|c|}
\hline Characteristics & $\begin{array}{l}\text { MLPA negative } \\
\mathrm{n}(\%)\end{array}$ & $\begin{array}{l}\text { MLPA positive } \\
\text { n (\%) }\end{array}$ & $P$-value \\
\hline Total & $294(80.0)$ & $74(20.0)$ & \\
\hline \multicolumn{4}{|l|}{ Gender } \\
\hline Male & $169(57.0)$ & $42(56.8)$ & \\
\hline Female & $125(43.0)$ & $32(43.2)$ & 0.999 \\
\hline \multicolumn{4}{|c|}{ Age range, (years) } \\
\hline$\leq 1$ & $15(5.1)$ & $0(0.0)$ & \\
\hline $2-10$ & $185(62.6)$ & $62(83.8)$ & \\
\hline$>10$ & $94(32.3)$ & $12(16.2)$ & $0.001^{* *}$ \\
\hline \multicolumn{4}{|l|}{ Skin color } \\
\hline White & $117(42.5)$ & $36(50.0)$ & \\
\hline Non-white & $159(57.5)$ & $36(50.0)$ & 0.300 \\
\hline \multicolumn{4}{|l|}{ WBC, $\times 10^{9} / 1$} \\
\hline Median & 61.1 & 44.8 & \\
\hline Range & $0.6-978.0$ & $2.2-95.0$ & \\
\hline$\leq 20$ & $153(52.8)$ & $40(54.8)$ & \\
\hline $20-50$ & $56(19.3)$ & 15 (20.5) & \\
\hline$>50$ & $81(27.9)$ & $18(24.7)$ & 0.816 \\
\hline \multicolumn{4}{|c|}{ Immunophenotype } \\
\hline Pro-B ALL & $31(10.5)$ & $1(1.4)$ & \\
\hline Common-ALL & $205(69.8)$ & $58(78.3)$ & \\
\hline Pre-B ALL & $58(19.7)$ & $15(20.3)$ & $0.038^{*}$ \\
\hline \multicolumn{4}{|l|}{$\mathrm{NCl}$ risk group } \\
\hline Standard risk & $141(48.3)$ & $46(63.0)$ & \\
\hline High risk & $151(51.7)$ & $27(37.0)$ & $0.018^{*}$ \\
\hline \multicolumn{4}{|l|}{ Status } \\
\hline Alive & $28(65.1)$ & $21(75.0)$ & \\
\hline Dead & $15(34.9)$ & $7(25.0)$ & 0.379 \\
\hline
\end{tabular}

$n$ number, $W B C$ white blood cell count $\times 10^{9} / /$ ${ }^{*} p<0.05 ;{ }^{* *} p \leq 0.01$ 
Patient material was first subjected to MLPA using the SALSA MLPA P327_A1 probe set. Relative peak heights between 0.75 and 1.3 were considered normal, while those below 0.75 and above 1.3 indicated losses or gains of genomic material, respectively (Fig. 1). Two hundred and ninety-four patients had no copy number changes of chromosome 21 regions since test fragments binding on chromosome 21 and control fragments binding on other chromosomes appeared normal, whereas in 74 patients gain of genetic material on chromosome 21 was found. None of our patients harbored gene deletions on chromosome 21.
For data validation and sub-division of MLPA positive patients we next applied the SALSA MLPA P327_B1 probe set. Down syndrome (DS) patients and cytogenetically confirmed hyperdiploid ALL with extra copies of chromosomes $21(\mathrm{Hyp}+21)$ were included as additional controls. We could not re-evaluate all 74 cases with chromosome 21 CNAs due to restrictions in the availability of DNA. However, for those patients subjected to analysis, no qualitative differences have been observed $(n=64)$; the presence or absence of CNAs on chromosome 21 has been confirmed. Pearson's correlation coefficients $\left(\mathrm{r}=0.09101, \mathrm{r}^{2}=0.8287, \mathrm{p}<0.0001\right)$, calculated to measure

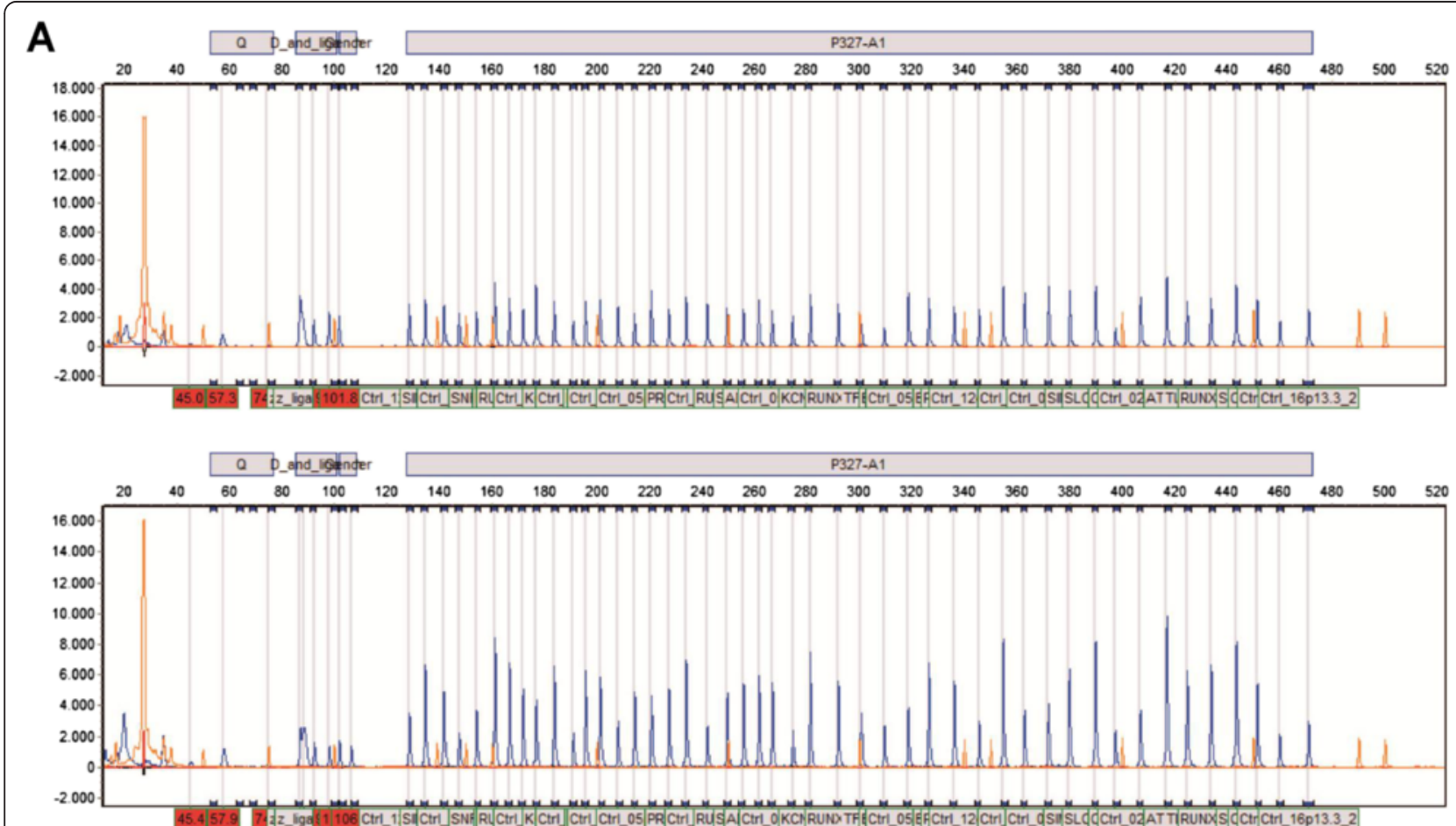

B
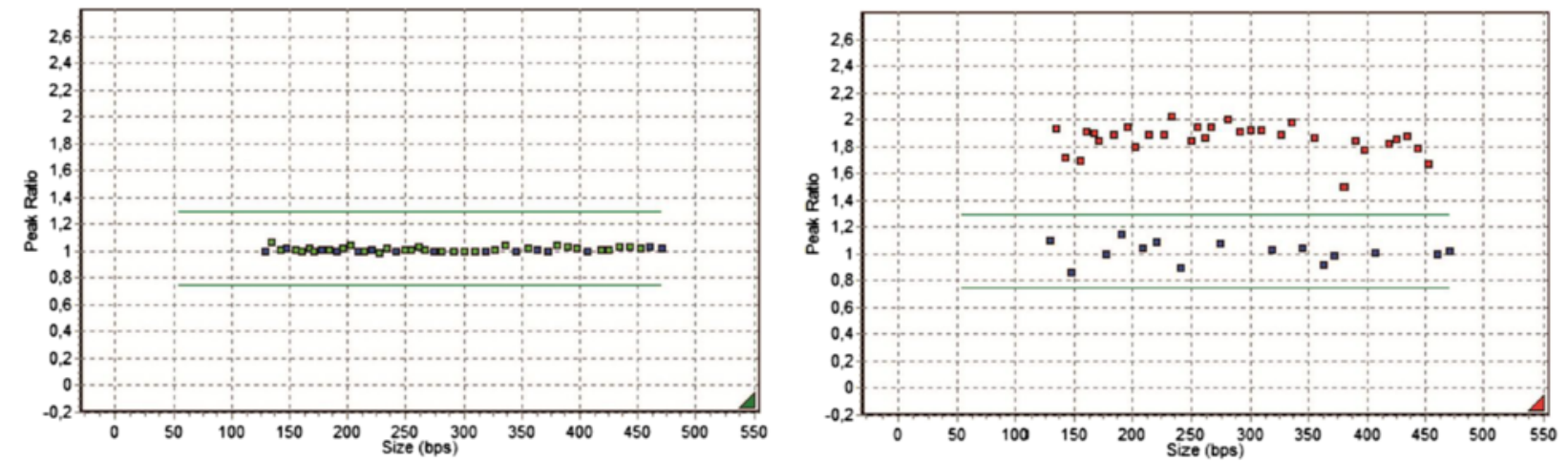

Fig. 1 Representative MLPA data shown as screen shots from the Gene-Marker 2.2 .0 analysis software. a. MLPA electropherograms of cord blood (CB) DNA (upper panel) compared to DNA from P4 (lower panel). Differences in peaks heights suggest altered dosages of corresponding exons depicted below. b. MLPA scatter plots for CB DNA (left panel) compared to P4 DNA (right panel). Reference probes (blue points) and test probes (green points) are within the normal range (located between green lines) for the CB sample, while test probes (red points) appear elevated for P4 
the linear dependence of variables and Kappa tests $(\kappa=0.661, \mathrm{SE}=0.083,95 \% \mathrm{CI}=0.498-0.825)$ to evaluate the number of observed agreements revealed only minor differences between average peak heights obtained from P327_A1 and P327_B1 probe sets (Fig. 2).

In order to understand gene CNAs distributions, MLPA results were illustrated in a heat map and arranged from centromeric to telomeric positions. MLPA peak heights allocated patients with chromosome 21 abnormalities into 3 main groups by a hierarchical clustering analysis (Fig. 3). Group 1 (bottom, $\mathrm{n}=8$ ) consisted of patients with slightly elevated MLPA peak ratios clustering in proximity to BCP-ALL without chromosome 21 CNAs; group 2 (middle, $\mathrm{n}=15$ ) of patients with intermediate peak ratios similar to those observed in DS and Hyp + 21 - and, group 3 (top, $n=26$ ) of patients with high or very high MLPA peak ratio profiles. Patient (P) 5 did not allocate into any of the three groups since its MLPA peak pattern demonstrated a higher variability than others.

According to availability of material FISH directed to the RUNX1 gene was performed in 50 patients. Six patients had a normal FISH pattern with 2 signals of RUNX1; in 35 patients 3-4 signals for RUNX1 - while in 9 patients $\geq 5$ signals for $R U N X 1$ were observed (Fig. 4a). Cells with 2-4 RUNX1 signals most likely acquired one or two additional copies of chromosome 21 (even though not detectable in all cases by interphase FISH), while those with $\geq 5$ RUNX1 signals fulfilled the internationally adopted criteria for an iAMP21.

Patients with an iAMP21 had a mean age of 55 months (range 22-120) and a median WBC (white blood cell count) of $15.9 \times 10^{9} / 1$ (range 7.2-92.0). According to National Cancer Institute (NCI) risk classification criteria, P5, P6 and P9 (33,3 \%) allocated to the high risk group. P1, P3, P4 and

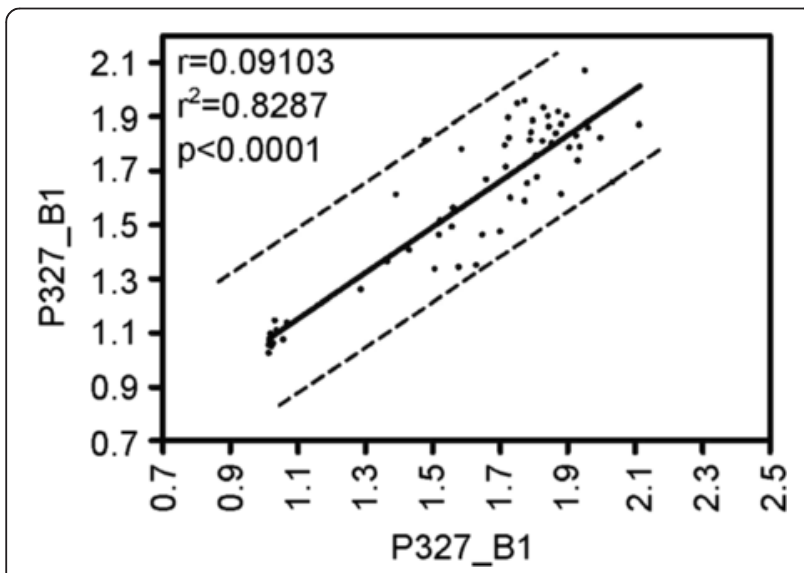

Fig. 2 Pearson's correlation coefficient analysis of data obtained from SALSA MLPA P327_A1 and P327_B1 probe sets. MLPA mean peak ratios over all chromosome 21 probes (black circles) were plotted for each patient; the black line depicts the linear correlation of data. Pearson correlation coefficients $(r), r$-squared $\left(r^{2}\right)$
P5 remained in complete remission until the last follow up in November 2014, while P8 and P9 succumbed to disease. For P2, P6, and P7 no follow up data was available.

For data comparison of the methodologies MLPA and FISH patients were clustered according to the number of RUNX1 signals (Fig. 4b). Mean MLPA peak ratios and standard deviations for chromosome 21 probes are provided in Additional file 1. MLPA peak ratios and in particular those of CRA genes separated cases with $\geq 5$ RUNX1 signals from cases with 2-4 RUNX1 signals. In the latter group these were lower and in the same magnitude as controls such as patients with DS (PT1-5) and Hyp + 21 (PH1-3) (Fig. 4c). We next performed a principal component analysis in order to illustrate the distribution of cases according to their MLPA peak profile. Patients were grouped into (1) FISH confirmed iAMP21, (2) $<5$ RUNX1 signals, (3) no FISH data, (4) DS and Hyp + 21, and (5) no chromosome 21 aberrations (Fig. 5). Sixtythree out of sixty-eight patients segregated into three clusters: The first one consisted of all patients from group 1 and two patients from group 3 and 4; the second of all patients from group 4 and most of the patients of group 2; and the third one of all patients from group 1 except P5. Interestingly, the latter cluster also contained some patients of group 2 and group 3 that located in close proximity to patients with an iAMP21; in other words exhibited an "iAMP21 like" MLPA peak profile.

Since we observed variations in MLPA peak heights between different patients, the correlation between RUNX1, DYRK1A, ETS2 and ERG gene load and expression was tested. Delta threshold cycle (dCT) values for each patient are shown in Table 2. For illustration we stepwise arranged cases from the lowest to the highest MLPA mean peak ratios and depicted corresponding dCT values (Fig. 6). Positive correlation between the MLPA mean peak ratios and $\mathrm{dCT}$ values were observed, since those patients with high MLPA mean peak ratios were the ones with low gene expression. This observation particularly held true for the genes RUNX1, DYRK1A, and ERG.

\section{Discussion}

In this study, we evaluated MLPA as a tool to detect an iAMP21 in a cohort of BCP-ALL, providing first data of this leukemia entity in a Brazilian BCP-ALL cohort. The information about gene CNA distributions on chromosome 21 was re-tested. Nine out of 368 BCP-ALL patients $(2.4 \%)$ were identified with an iAMP21 according to criteria specified recently [13]. Taking submicroscopic aberrations into account examined by MLPA, additional cases presenting high MLPA peak ratios of genes within the CRA region or exhibiting an "iAMP21 like" peak ratio profile of chromosome 21 genes might be considered as positive for this chromosomal abnormality and subjected to further genomic tests. 


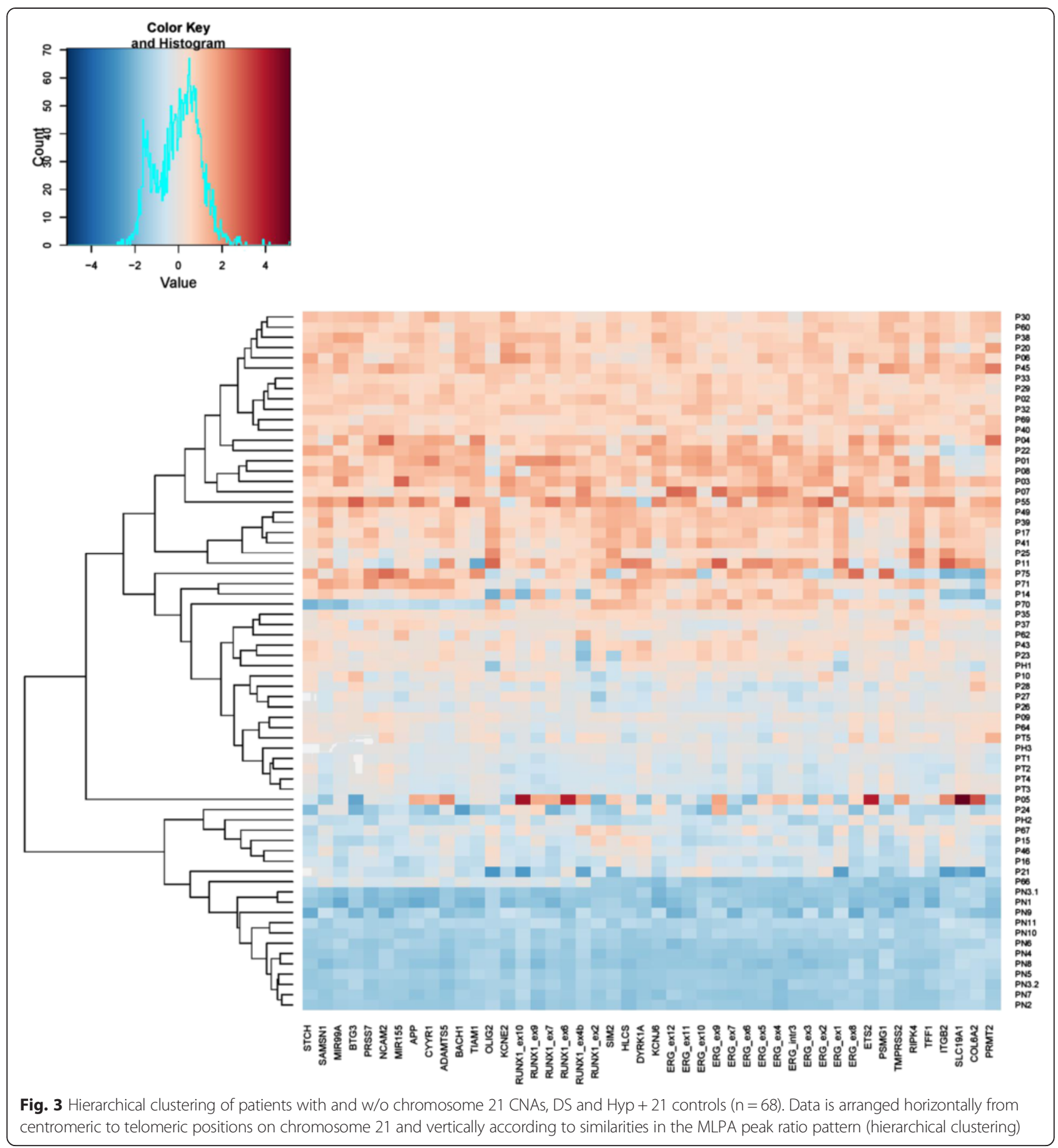

In the past 10 years MLPA was successfully applied as a tool in cancer research, providing precise information on increased or decreased copy numbers at specific loci [14]. More recently it has been proven as accurate and sensitive for detection of CNAs in hematological malignancies, particularly appearing advantageous for analyzing large sample sets in a time efficient fashion $[15,16]$. MLPA demonstrated comparable detection rates to routine interphase FISH for aberrations being typically present in leukemia. Since it can detect also rare chromosomal aberrations it was suggested being applied as an initial test if routine cytogenetics is not possible or informative [17]. The SALSA P327_A1 and P327_B1 probe sets were designed to provide information on the copy number status of 24 and 29 genes located on chromosome 21, respectively. Six of the 31 probes targeting chromosome 21 are specific for RUNX1 gene being located within the CRA. Other probes anneal to 


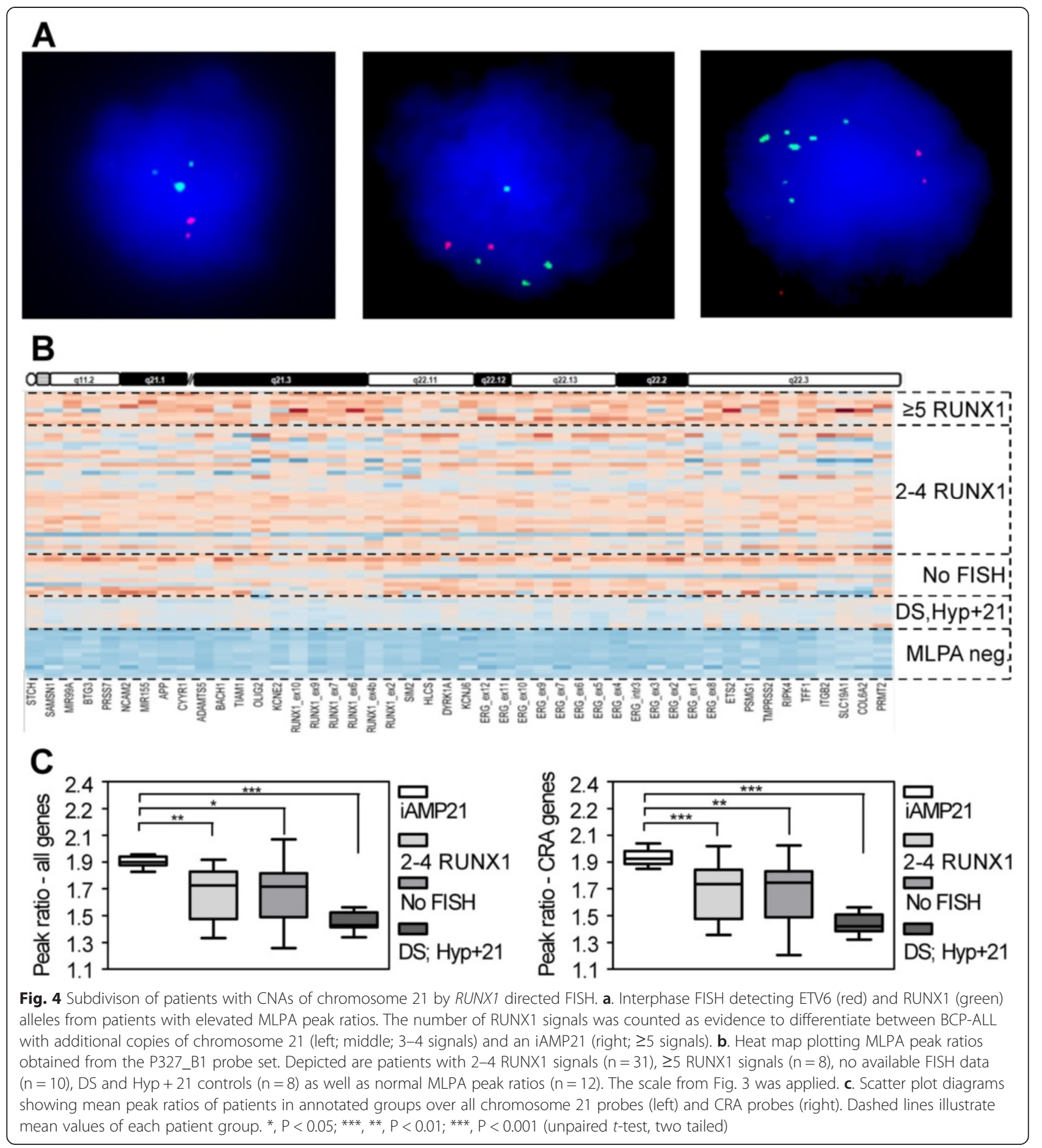

regions flanking the RUNX1 gene described being duplicated or amplified in most cases with an iAMP21. In our hands low MLPA mean peak ratios within the CRA $(<1.8)$ ruled the presence of an iAMP21 out, in all of these cases less than three additional RUNX1 signals have been detected. By contrast, high MLPA mean peak $(\geq 1.8)$ ratios should be taken as evidence for the presence of an iAMP21, even though encountering difficulties to detect this chromosomal abnormality by FISH performed from interphase preparations. Nevertheless, we cannot rule out that MLPA in some cases fails to accurately distinguish between BCP-ALL with 4 copies of a chromosome 21 and an iAMP21 due to certain variability between blast counts of different patient samples. On the other hand interphase FISH could not detect additional RUNX1 signals in a total of six cases, shown to have gain of genetic material by 


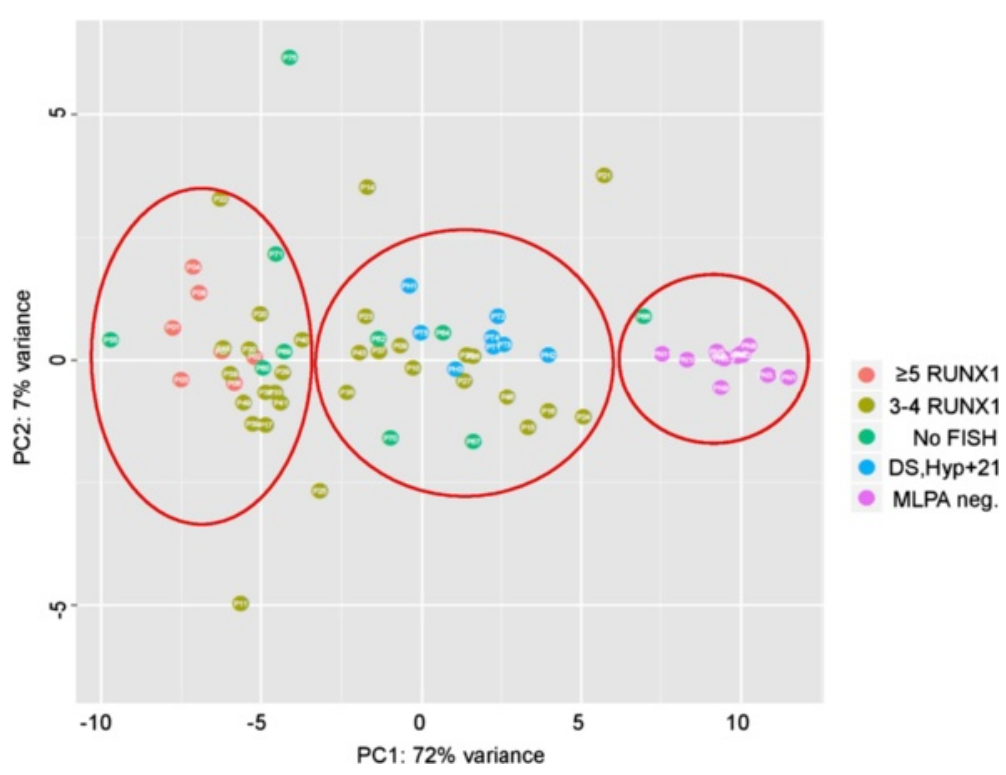

Fig. 5 Principal component analysis of grouped patients. Individuals are distributed according to similarities in their MLPA peak ratio profiles and depicted by color-coded circles that designate certain patient groups. Red Circles demonstrate the presence of three main clusters

MLPA. Even though we are aware of such methodological pitfalls, these cases unlikely harbored an iAMP21 since peak ratios obtained from both data sets were low to intermediate. Further investigation of such cases by whole genome techniques such as array based comparative genomic hybridization or, alternatively exome sequencing capable of assessing gene copy numbers would be desirable.

Depending on the chromosomal location of genes, MLPA peak ratios appeared heterogeneous in BCP-ALL with an iAMP21. The highest MLPA peak ratios and lowest variations between different patients were encountered within and close to the CRA while peak ratios often dropped towards telomeric regions. These observations are in line with the view that chromosome 21 aberrations in BCP-ALL with an AMP21 are highly complex, exhibiting a stepwise increase in copy numbers towards the CRA and decrease of copy number towards the CRD,

Table 2 RT-qPCR data of patients with an iAMP21 for selected genes located in the CRA

\begin{tabular}{lcccc}
\hline$\#$ & dCT DYRK1A & dCT ERG & dCT ETS2 & dCT RUNX1 \\
\hline P1 & 8.715 & 1.790 & 0.555 & 5.950 \\
P2 & 11.625 & 7.655 & 6.430 & 12.195 \\
P3 & 3.995 & 1.775 & 1.760 & 3.515 \\
P4 & 7.885 & 5.380 & 4.155 & 7.250 \\
P5 & 9.905 & 7.230 & 6.200 & 12.595 \\
P6 & 9.815 & 6.665 & 6.200 & 11.095 \\
P7 & 8.420 & 6.765 & 3.895 & 9.630 \\
P8 & 11.010 & 5.635 & 4.845 & 11.000 \\
\hline
\end{tabular}

mapping centromeric and telomeric parts of chromosome 21 , respectively [10].

Due to the lack of studies identifying individual genes, playing an important role in leukemogenesis or providing evidence that de-regulation of certain candidates would explain the poor outcome of patients with an iAMP21, the effect of gene load on mRNA expression within the CRA was also evaluated. In contrast to our expectations, this analysis revealed that patients with lowest gene dosages of RUNX1, DYRK1A, ETS2 and ERG genes displayed the highest expression of the respective mRNA. These results need to be consolidated analyzing more patients. As part of a study to describe genomic alterations and gene expression in BCP-ALL with an iAMP21, Strefford et al. 2006 evaluated the expression of genes located in the CRA of eight cases and compared data with that of other BCP-ALL subtypes. Even though expression levels of the genes contained within the CRA were higher in BCP-ALL with an iAMP21 for almost half of the probe sets from the CRA no over-expression was observed, including the RUNX1 gene. The authors concluded that these results could be explained by regulatory mechanisms such as epigenetics and biofeedback in BCP-ALL [9]. Strikingly, hyperdiploid leukemias, by definition harboring less gene copies on chromosome 21 than cases with an iAMP21, showed higher mRNA expression for the genes analyzed.

\section{Conclusions}

Altogether, we confirmed that aberrations on chromosome 21 in BCP-ALL with an BAMP21 represent a high level of complexity but as a group exhibited similar MLPA peak ratio profiles and, furthermore provided evidence for 


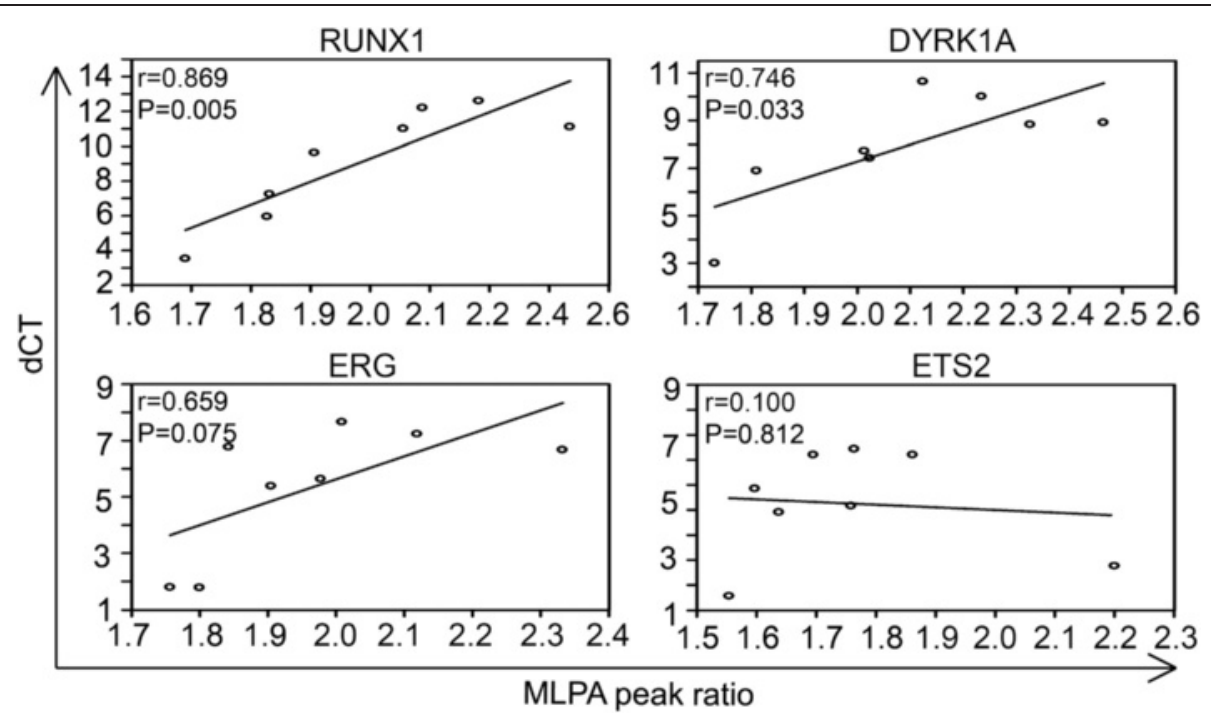

Fig. 6 Correlation of MLPA mean peak ratios and gene expression in patients with an iAMP21. Scatter plots demonstrate the relation between mean MLPA peak ratios and mRNA expression of genes located within the CRA. Each circle represents data of one individual. Similarities between variables are depicted by linear regression (lines), Pearson correlation coefficients ( $r$ ) and p-values

the existence of gene regulatory mechanisms that might modulate the expression of amplified genes. MLPA profiles of CRA genes might be taken into account to facilitate diagnosis whenever metaphase nuclei for FISH are not available. However, for identification of an iAMP21 solely by MLPA next generation probe sets would need to include control fragments binding genes on chromosomes usually over-represented in hyperdiploid ALL.

\section{Methods}

\section{Patients}

A series of consecutive diagnostic samples from Brazilian childhood BCP-ALL patients collected between the years 2002 to 2012 were selected according to availability of material of good quality providing that bone marrow aspirates harbored at least $30 \%$ of blast cells. The initial dataset contained samples from 606 patients. Out of those, 368 samples were retained for this study, excluding 238 cases based on the available results of mutually exclusive gene fusions (ETV6/RUNX1, TCF3/PBX1, MLL-r and $B C R-A B L 1)$. In addition five patients with ALL and Down syndrome were included.

As criteria for assigning as standard-risk or high-risk patients, age at diagnosis and WBC according to the Cancer Therapy Evaluation Program of the NCI were considered. Data collection and laboratory procedures have been evaluated and approved by the Research Ethics Committee from the National Cancer Institute (INCA) in Rio de Janeiro (CEP \#005/06 and CAAE 626.268 at April 28, 2014). Informed consent was obtained from all subjects or their parents in accordance with the Declaration of Helsinki.

\section{Multiplex ligation-dependent probe amplification}

The presence of an iAMP21 has been evaluated applying the MLPA SALSA P327_A1 (lot A1-0101) and P327_B1 (lot B1-0613) probe sets (MRC Holland, Amsterdam, The Netherlands). Tests fragments have been designed to evaluate the copy number status of genes distributed from centromeric to telomeric regions (q11.2-q22.3, 14.668-46.356) of chromosome 21. The P327_B1 probe set represents a modified version of the P327_A1 probe set in which 12 new probes for the $E R G$ gene and 3 additional probes for chromosome 21 genes have been added. Four RUNX1 specific probes (exon 10; 7;6 and 4b) have been replaced while two probes (exon 9 and 2) remained unchanged. The size and location of most other test probes as well as reference probes has been redefined. Detailed information about P327_A1 and P327_B1 MLPA probe sets was downloaded from the MRC Holland website (http://www.mlpa.com); see Additional files 2 and 3, respectively. MLPA and capillary electrophoresis based amplification product separation (ABI 3130xl, Life Technologies, Carlsbad, CA) was performed according to the manufacturer's instructions. Relative copy numbers were obtained after normalization of peaks against cord blood derived controls. Sequences were analyzed by the Gene Marker 2.2.0 software (Soft Genetics LLC, State College, PA).

\section{Fluorescence in situ Hybridization (FISH)}

FISH was performed using the "LPH012 TEL/AML1 Translocation, Dual Fusion Probe", designed to detect the TEL/AML1 fusion gene. Cells were processed 
according to the protocols instructions (Cytocell, Cambridge, UK). Cases were considered positive for an iAMP21 when $\geq 5$ RUNX1 signals were observed in at least $7 \%$ of metaphase nuclei (standard deviation +/$2 \%$ ). If metaphase FISH was not possible, an iAMP21 was identified as multiple copies of RUNX1 in most interphase nuclei $[1,13]$.

\section{Reverse trancriptase quantitative (RT-q)PCR}

Total RNA was isolated by Trizol reagent (Life Technologies, Carlsbad, CA) and used for cDNA synthesis by Super Script II Reverse Transcriptase (Life Technologies, Carlsbad, CA). RUNX1, ETS2, DYRK1A, ERG and, GAPDH transcripts used as an endogenous control were quantified by SYBR-green RT-qPCR (Bioline, London, UK) using the Applied Biosystems 7300 Real-Time PCR System (Applied Biosystems, Foster City, CA). For data depiction dCT values (CT target - CT reference) of biological replicates were calculated. The following primer combinations were used: RUNX1, forward 5'-GAGCTGAGAAATGCTA CCGC-3', reverse 5'-GGTCAGAGTGAAGCTTTTCCC-3'; ETS2, forward 5'-TTCCAAAGAACCCCTGGCTG-3', reverse 5'-CGAACCTCTGCAGATTCACG-3'; DYRK1A, forward 5'- TGTAACCCCAAACGCAGTG-3', reverse 5'- ACCGATAAAAGCGACTCTGAA-3'; ERG, forward 5'-AAGTAGCCGCCTTGCAAAT-3', reverse 5'-GTGC CTTCCCAGGTGATG-3'; and GAPDH, forward 5'-TGA CCCCTTCATTGACCTCA-3', reverse 5'-AGTCCTTCC ACGATACCAAA-3'.

\section{Statistical analysis}

Statistical analyses were performed using the Statistical Package for Social Sciences (SPSS), 18 (Chicago, IL), Graph Pad Prism 4.02 (La Jolla, Ca) and R (Foundation for Statistical Computing, Auckland, New Zealand) software packages. Confidence intervals of $95 \%$ were used; $P$-values $<0.05$ were considered statistically significant. Chi-square and Fisher's exact tests were used for comparative variable analyzes of demographic and clinical variables. Correlation and the strength of agreement between MLPA data were assessed by Pearson correlation coefficients and Kappa tests, respectively. Hierarchical clustering and principal component analyses (PCA) were created in R. For creation of heat maps MLPA values were scaled and centered using the scale function followed by clustering and plotting of normalized values using heat map .2 with default values. Patient groups from heatmaps were compared by unpaired, two sided Student's T-tests. PCA was performed on scaled values using the $\operatorname{prcomp}()$ function with default parameters.

\section{Additional files} Additional file 1: Mean MLPA peak ratios in BCP-ALL obtained
from test fragments of the MLPA SALSA P327-B1 probe set.

Additional file 2: The size and location of each test and reference fragment of the MLPA SALSA P327_A1 probe set.

Additional file 3: The size and location of each test and reference fragment of the MLPA SALSA P327_B1 probe set.

\section{Abbreviations}

BCP-ALL: B-cell precursor acute lymphoblastic leukemia; CNAs: Copy number alterations; CRA: Common region of amplification; CRD: Common region of deletion; dCT: Delta threshold cycle; DS: Down syndrome; FISH: Fluorescence in situ hybridization; Hyp + 21: Hyperdiploid ALL with extra copies of chromosomes 21; iAMP21: Intrachromosomal amplification of chromosome 21; P: Patient; PCA: Principal component analysis; PH: Patients with Hyp + 21; PT: Patients with DS; WBC: White blood cell count.

\section{Competing interests}

The authors declare that they have no competing interests.

\section{Authors' contributions}

ETGP performed diagnostic immunphenotyping of BCP-ALL cases. GF and TCB carried out and analyzed MLPA data. LH, CB and TMFV performed FISH. ME assisted in interpretation of gene expression data acquired by JCS. GF designed the study, interpreted data and wrote the manuscript. MSPO was the principal investigator, coordinated the research, assisted in data interpretation and edited the manuscript together with ME.

All authors read and approved the final manuscript.

\section{Acknowledgments}

The authors would like to thank for Alessandra Faro, Bruno Alves Aguiar Gonçalves, Camilla Andrade and Dr. Gisele Vasconcelos for technical support. We particularly appreciate all bioinformatical assistance of Dr. Christian Frech from the Children's Cancer Research Institute in Vienna, Austria. MSPO has been supported by CNPq Research Scholarships \#302423/2010-9 and FAPERJ \#E026/101.562/2010. The Brazilian Ministry of Health supported GF through the Institutional Development Scholarship Program.

Received: 22 April 2015 Accepted: 20 May 2015

Published online: 10 June 2015

\section{References}

1. Robinson HM, Broadfield ZJ, Cheung KL, Harewood L, Harris RL, Jalali GR, et al. Amplification of AML1 in acute lymphoblastic leukemia is associated with a poor outcome. Leukemia. 2003;17(11):2249-50.

2. Harewood L, Robinson H, Harris R, Al-Obaidi MJ, Jalali GR, Martineau M, et al. Amplification of AML1 on a duplicated chromosome 21 in acute lymphoblastic leukemia: a study of 20 cases. Leukemia. 2003;17(3):547-53.

3. Moorman AV, Richards SM, Robinson HM, Strefford JC, Gibson BE, Kinsey SE, et al. Prognosis of children with acute lymphoblastic leukemia (ALL) and intrachromosomal amplification of chromosome 21 (iAMP21). Blood. 2007;109(6):2327-30.

4. Heerema NA, Carroll AJ, Devidas M, Loh ML, Borowitz MJ, Gastier-Foster JM, et al. Intrachromosomal amplification of chromosome 21 is associated with inferior outcomes in children with acute lymphoblastic leukemia treated in contemporary standard-risk children's oncology group studies: a report from the children's oncology group. J Clin Oncol. 2013;31(27):3397-402.

5. Moorman AV, Robinson H, Schwab C, Richards SM, Hancock J, Mitchell CD, et al. Risk-directed treatment intensification significantly reduces the risk of relapse among children and adolescents with acute lymphoblastic leukemia and intrachromosomal amplification of chromosome 21: a comparison of the MRC ALL97/99 and UKALL2003 trials. J Clin Oncol. 2013;31(27):3389-96.

6. Attarbaschi A, Panzer-Grumayer R, Mann G, Moricke A, Konig M, Mecklenbrauker A, et al. Minimal residual disease-based treatment is adequate for relapse-prone childhood acute lymphoblastic leukemia with an intrachromosomal amplification of chromosome 21: the experience of the ALL-BFM 2000 trial. Klin Padiatr. 2014;226(6-7):338-43. 
7. Harrison CJ, Moorman AV, Schwab C, Carroll AJ, Raetz EA, Devidas M, et al, An international study of intrachromosomal amplification of chromosome 21 (iAMP21): cytogenetic characterization and outcome. Leukemia. 2014;28(5):1015-21.

8. Baldus CD, Liyanarachchi S, Mrozek K, Auer H, Tanner SM, Guimond M, et al. Acute myeloid leukemia with complex karyotypes and abnormal chromosome 21: Amplification discloses overexpression of APP, ETS2, and ERG genes. Proc Natl Acad Sci U S A. 2004;101(11):3915-20.

9. Strefford JC, van Delft FW, Robinson HM, Worley H, Yiannikouris O, Selzer R, et al. Complex genomic alterations and gene expression in acute lymphoblastic leukemia with intrachromosomal amplification of chromosome 21. Proc Nat Acad Sci U S A. 2006;103(21):8167-72.

10. Rand V, Parker H, Russell L, Schwab C, Ensor H, Irving J, et al. Genomic characterization implicates IAMP21 as a likely primary genetic event in childhood B-cell precursor acute lymphoblastic leukemia. Blood. 2011;117(25):6848-55.

11. Li Y, Schwab C, Ryan SL, Papaemmanuil E, Robinson HM, Jacobs P, et al. Constitutional and somatic rearrangement of chromosome 21 in acute lymphoblastic leukaemia. Nature. 2014;508(7494):98-102.

12. Robinson HM, Harrison CJ, Moorman AV, Chudoba I, Strefford JC. Intrachromosomal amplification of chromosome 21 (iAMP21) may arise from a breakage-fusion-bridge cycle. Genes Chromosomes Cancer. 2007:46(4):318-26.

13. Harrison CJ. Blood Spotlight on iAMP21 acute lymphoblastic leukemia (ALL), a high-risk pediatric disease. Blood. 2015;125(9):1383-6.

14. Schouten JP, McElgunn CJ, Waaijer R, Zwijnenburg D, Diepvens F, Pals G Relative quantification of 40 nucleic acid sequences by multiplex ligation-dependent probe amplification. Nucleic Acids Res. 2002;30(12):e57.

15. Schwab CJ, Jones LR, Morrison H, Ryan SL, Yigittop H, Schouten JP, et al. Evaluation of multiplex ligation-dependent probe amplification as a method for the detection of copy number abnormalities in B-cell precursor acute lymphoblastic leukemia. Genes Chromosomes Cancer. 2010;49(12):1104-13.

16. Konialis C, Savola S, Karapanou S, Markaki A, Karabela M, Polychronopoulou S, et al. Routine application of a novel MLPA-based first-line screening test uncovers clinically relevant copy number aberrations in haematological malignancies undetectable by conventional cytogenetics. Hematology. 2014;19(4):217-24.

17. Alhourani E, Rincic M, Othman MA, Pohle B, Schlie C, Glaser A, et al. Comprehensive chronic lymphocytic leukemia diagnostics by combined multiplex ligation dependent probe amplification (MLPA) and interphase fluorescence in situ hybridization (iFISH). Mol Cytogenet. 2014;7(1):79.

\section{Submit your next manuscript to BioMed Central and take full advantage of:}

- Convenient online submission

- Thorough peer review

- No space constraints or color figure charges

- Immediate publication on acceptance

- Inclusion in PubMed, CAS, Scopus and Google Scholar

- Research which is freely available for redistribution 